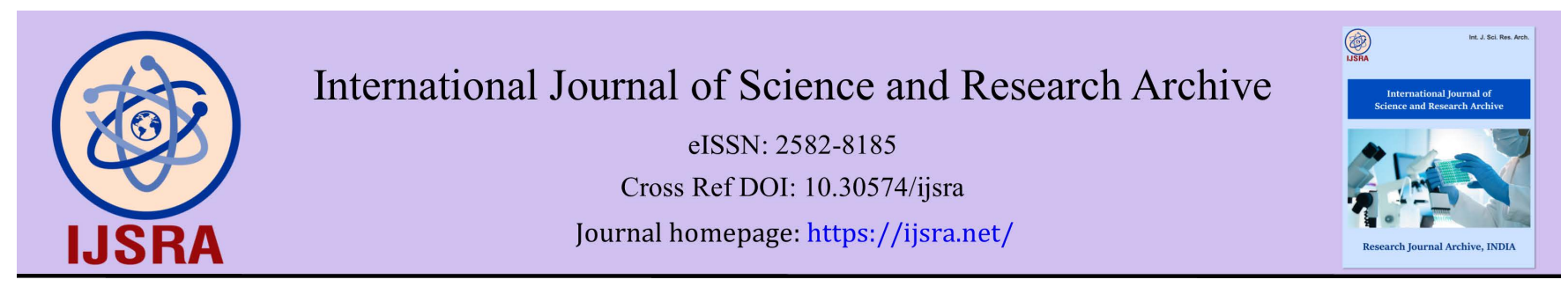

(REVIEW ARTICLE)

\title{
Oral exfoliative cytology - A technical appraisal in oral diseases
}

\author{
Sreeshyla HS ${ }^{1,}{ }^{*}$, Hegde Usha ${ }^{1}$, Srinivas J ${ }^{2}$, Priyanka Nitin ${ }^{1}$ and Premalatha BR ${ }^{1}$ \\ ${ }^{1}$ Department of Oral Pathology \& Microbiology, JSS Dental College \& Hospital, A Constituent College of JSS AHER, Mysuru. \\ Karnataka, India. \\ 2 Department of Oral Pathology \& Microbiology, Poorvanchal Institute of Dental Sciences, Gorakhpur, Uttar Pradesh.
}

International Journal of Science and Research Archive, 2021, 04(01), 115-118

Publication history: Received on 17 October 2021; revised on 01 December 2021; accepted on 03 December 2021

Article DOI: https://doi.org/10.30574/ijsra.2021.4.1.0184

\begin{abstract}
Oral exfoliative cytology is one of the easy, simple, economical and non-cumbersome diagnostic adjunct. It involves examining the exfoliated buccal cells for alterations in cellular and nuclear features. It offers the advantage of being useful in less resource setting areas. Though associated with many false results, the newer technological advancements have proven its diagnostic efficiency. Oral exfoliative cytology can readily be used as adjunct to disease diagnosis, however, it cannot replace biopsy.
\end{abstract}

Keywords: Exfoliative cytology; Oral disease; Cancer; Diagnosis; Technique; Cell

\section{Introduction}

Exfoliative cytology is one of the most common diagnostic adjunct adapted in the disease diagnosis. With the success of cytology in cervical pathologies, it was adopted to other non cervical pathologies including oral lesions. It was used for studying various oral diseases, especially as an aid in the early detection of oral precancer and oral cancer [1]. Currently the technique has advanced vastly to involve DNA cytomorphometry, quantitative analysis, identification of tumor markers and other molecular studies [2,3].

\subsection{Definition}

Exfoliative cytology is the microscopic examination of shed or desquamated cells from an epithelial surface. It is used as means of detecting malignancy and microbiologic changes and to measure hormonal levels. Such cells for examination can be obtained by scraping, aspiration, washing or smears. It is a rapid, non-invasive, economical procedure. Its principle use is in screening oral diseases, mainly the oral cancer $[4,5,6]$.

Oral exfoliative cytology initially was used to study salivary cellular contents in patients with dental caries. However, later studies were on the normal process of epithelial keratinization and keratinization in certain mucocutaneous conditions such as leukoplakia, lupus erythematosus and lichen planus. Introduction of PAP was an added advantage. The main basis for cytological diagnosis in various malignancies was based on the work of Papanicolaou and Traut, who introduced novel techniques for collecting and staining cells for the diagnosis of the disease $[4,5]$.

In 1843 Walsh identified and described cancer cells in patient's sputum. Cytological diagnosis in a pharyngeal carcinoma case using sputum sample was performed by Bealem in 1860. Ziskin, in 1941 is the first person to use exfoliative cytology in oral cavity for disease reporting. Subsequently oral cytology was used by several investigators to study and

\footnotetext{
${ }^{*}$ Corresponding author: Sreeshyla HS

Department of Oral Pathology \& Microbiology, JSS Dental College \& Hospital, A Constituent College of JSS AHER, Mysuru. Karnataka, India.

Copyright $(2021$ Author(s) retain the copyright of this article. This article is published under the terms of the Creative Commons Attribution Liscense 4.0.
} 
examine the normal oral cellular keratinization and oral conditions. Cytological diagnosis of oral cancer was performed by Ziskin Montgomery and Von Hamm in 1951 [2,3]. Oral cytology sample can be collected using conventional oral brush, wooden spatula, cotton-tipped wooden spatula and a metal spatula. Studies have shown a sensitivity and specificity of 78.6-100\% with these instruments [2-6].

\section{Basis of exfoliative cytology}

The regular physiological process of keratinization and desquamation, forms the basis for exfoliative cytology. During the process of keratinization, as part of normal physiologic turnover the squamous cells of the oral epithelium show regular exfoliation of its superficial cells. Under normal conditions, the cells in the deeper layer are strongly attached to each other through the cell junctions. However, some of these cells loose their cohesiveness under some pathological conditions, leading to exfoliation of some of the deeper cells along with the superficial cells. These exfoliated cells are collected using specific instruments and are studied under the microscope [1,3,7].

When diseased, the cells in the epithelium show certain cellular and nuclear changes. The dysplastic epithelium involved with premalignant and malignant diseases show these changes prominently. These cytological features include increased nuclear cytoplasmic ratio, variations in size and shape of the cell and its nucleus, nuclear discontinuity, mitotic figures, texture of the nucleus and micronuclei. These cytological alterations studied under microscope using exfoliative cytology aids in specific disease diagnosis. It is also possible to analyze these cells both quantitatively and qualitatively [5].

\subsection{Indications $[4,5,6,9]$}

- Cytology can be used as an adjunct diagnostic aid to biopsy in the diagnosis of oral lesions

- Determination of suitable site for biopsy

- Periodic review of oral potentially malignant disorders and oral cancer

- Periodic examination of high risk patients, such as the persons with the habit of tobacco, alcohol or betel quid.

- Under compromised conditions when biopsy is contraindicated

- Unavailability of resources for processing of biopsy

- Mass screening of population for oral cancer

- Diagnosis of oral mucosal lesions such as herpes infections, pemphigus, keratosis follicularis, intraepithelial dyskeratosis, white sponge nevus and protein-calorie malnutrition deficiency by the presence of certain specific cells.

- For detection of sex chromosomes

- Detection of oral microorganisms

- To study cellular and nuclear changes in various conditions such as anemia, pernicious anemia, tropical sprue, diabetes etc.

- Research applications

- In forensic odontology

\subsection{Contraindications $[4,5,9]$}

- Deep seated lesions

- Fibrous growths

- Polypoid lesions

- Non-ulcerative conditions

- Atropic lesions

- Densely keratinized lesions

- Smooth surface lesions

- Patients with underlying blood dyscrasias

\subsection{Technique $[4,5,6]$}

Instruments required -oral cytology brush/ wooden/metal spatula, slides, fixative 


\subsection{Procedure}

- Remove the debris and mucin from the surface of the oral lesion.

- Collect the material from the lesion by using slight rolling and scraping motion using the cytobrush or cotton applicator or wooden spatula

- If the area to be scraped is dry, the applicator or the spatula should be moistened.

- Smears may be inadequate if there is a pseudomembrane covering a lesion, thick saliva, excess bleeding or no moisture.

- The collected material is spread uniformly over a microscopic slide and fixed immediately before the smear dries. A monolayer of cells is preferred after a smear preparation.

- The smear is stained using appropriate staining technique.

- Cleared in xylene and mounted for microscopic examination.

$70 \%$ alcohol is adequate for fixation. Commercial spray fixitives can also be used. The smear can be stained using routine hematoxylin and eosin, Papanicolaou (PAP) stain, periodic acid Schiff(PAP) or any other special stains.

\subsection{Interpretation}

When stained under PAP, the epithelial cells show specific colours such as orange, pink, blue green or green colour depending on the surface layer it belongs to [5].

\section{Appearance of surface cells in relation to location [9]}

Hard palate, gingiva, dorsal tongue - mature cells, high degree of cornification and some anucleated cells. Buccal and labial mucosa - intermediate maturity, nucleated basophilic and acidophilic staining cells. Floor of mouth, ventral tongue, soft palate, oropharynx - least maturity and preponderantly basophilic cells with large nuclei

Features of the smear that are diagnostic of oral premalignant and malignant lesions are increased keratinization, increased nuclear area, increased nuclear-cytoplasmic ratio, nuclear hyperchromatism, nuclear pleomorphism, prominent and irregular nucleus, multiple prominent and irregular nucleoli, abnormal chromatin pattern and distribution and discrepancy in maturation

Accurate cytological interpretation of the cellular smear depends on several factors such as methods of collection of the cytological specimen, adequacy of the specimen, fixation and fixative solutions used, preservation of the cytological specimen prior to processing, smear preparation for microscopic examination, staining and mounting of the smear.

Depending on the cellular and nuclear features, the cytologic smears of oral potentially malignant disorders and oral cancer will be reported to be in the category of Class I to Class V $[4,6,9]$. Class -I indicates normal, Class - II Atypical, Class - III Intermediate, Class -IV Suggestive of cancer and Class - V Positive for cancer

The cytosmear can also be reported as Negative for cancer similar to Class I and Class II, Suggestive or intermediate similar to Class III and Class IV, Positive for cancer similar to Class V and Inadequate [6,9].

\subsection{Advantages}

- Painless and bloodless non-invasive procedure

- It is quick and simple

- Suitable in medically compromised patients, contraindicated for biopsy

- Safeguards against false negative biopsy

- Elimination of post biopsy complications

- Easy, reliable and most feasible technique

\subsection{Disadvantages}

- Provides moderately lesser information when compared to a histological slide. Only the individual cells, surface epithelial cells are obtained and cannot be studied in their proper tissue relationship to one another. 
- Negative report will not rule out cancer. Biopsy is indicated for final confirmation in all the clinically suspicious lesions.

- Some oral disorders cannot be diagnosed cytologically because of the scarcity of exfoliated surface cells.

- Appropriate only for epithelial lesions or for tissues with exfoliating cells and is rarely useful in the evaluation of connective tissue lesions.

- It can only be an adjuvant, but not a substitute for the biopsy.

- Presence of an invasion and its extent cannot be assessed.

- Tumour grading cannot be assessed.

- Interpretation requires an experienced cytopathologist.

\section{Conclusion}

Oral exfoliative cytology is a simple, quick, painless diagnostic procedure. It can act as an adjunct to histopathological diagnosis, but can never replace a biopsy. Despite the numerous studies on oral exfoliative cytology, the applicability and reliability of the procedure is still debatable. The diagnostic accuracy of the cytosmear is predicted upon the proper smear preparation and sufficient experience in its evaluation by the pathologist.

\section{Compliance with ethical standards}

\section{Disclosure of conflict of interest}

No conflict of interest.

\section{References}

[1] Folsom TC, White CP, Bromer L, Canby HF, Garrington GE. Oral exfoliative cytology. Review of the literature and report of a three-year study. Oral Surg. 1972; 33(1): 61-74.

[2] Mehrotra R, Hullmann M, Smeets R, Reichert TE, Driemel O. Oral cytology revisited. J Oral Pathol Med. 2009; 38: 161-166.

[3] Sivapathasundharam B, Kalasagar M. Yet another article on exfoliative cytology. J Oral Maxillofac Pathol. 2004; 8: 54-7.

[4] Das BK, Mallick NC. The diagnostic perspectives of oral exfoliative cytology - an overview. JIDA. 2000; 71: 7-9.

[5] Sugerman PB, Savage NW. Exfoliative cytology in clinical oral pathology. Australian Dental Journal. 1996; 41: 7174.

[6] Bernstein ML, Miller RL. Oral exfoliative cytology. JIDA. 1978; 96: 625-629.

[7] Sreeshyla HS, Shashidara R, Sudheendra US. Diagnostic aids in oral precancer and cancer. Indian Journal of Multidisciplinary Dentistry. 2014; 4(2): 928-934.

[8] Burkhardt A. Advanced methods in the evaluation of premalignant lesions and carcinomas of the oral mucosa. J Oral Pathol. 1985; 14: 751-778.

[9] Silverman S. American cancer society Atlas of Oral Oncology. Oral cancer. 5th edition. London. BC Decker Inc. 